\title{
Are children among the poorest?
}

\section{Ana Vaz, OPHI Researcher}

One recurrent question when presenting the MPI 2014 results is: and what about the children? To address this directly we could construct a child poverty measure, using indicators that capture the specific deprivations experienced by children and that are present in the DHS and MICS questionnaires. In fact, that work is already underway. ${ }^{\text {i }}$ Yet we could also ask how many children live in a household that is multidimensionally poor according to the Global Multidimensional Poverty Index (MPI) estimated by OPHI. The MPI includes indicators related directly with children (like child's nutrition and school attendance), but it is primarily a measure of poverty defined at the household level. So while the MPI does not focus on child poverty, we can observe how children are represented in multidimensionally poor households.

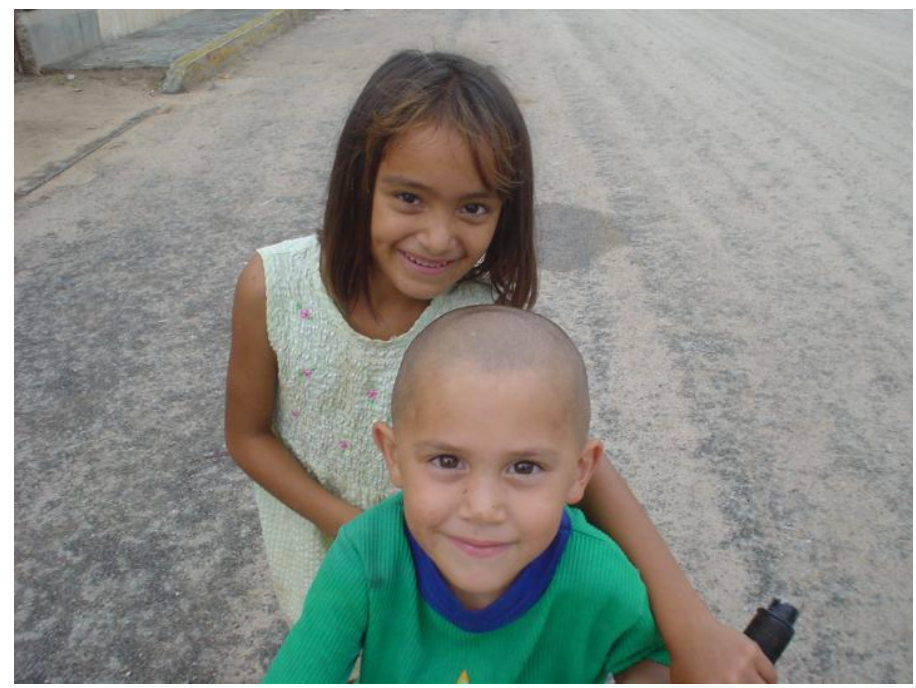

\section{What is the Global MPI?}

- The Global Multidimensional Poverty Index (MPI) is an index of acute multidimensional poverty that assesses the nature and intensity of poverty at the individual level, by directly measuring the overlapping deprivations poor people experience simultaneously.

- The $2014 \mathrm{MPI}$ covers 108 countries and $\mathbf{5 . 4}$ billion people, disaggregated by 780 subnational regions. Changes over time are reported for 2.5 billion people. OPHI's databank is fully downloadable and provides a wealth of consistent statistics.

- The Global MPI covers 10 indicators of health, education and living standards. For details, see www.ophi.org.uk/multidimensional-poverty-index

- A person is multidimensionally poor if they are deprived in at least one third of the dimensions.

- The MPI is calculated by multiplying the incidence of poverty by the average intensity of poverty across the poor; as a result it reflects both the share of people in poverty and the degree to which they are deprived.

Using a sample of countries for which MPI was recently updated, this note compares the age structure of the population and the age structure of the poor. ${ }^{\text {ii }}$ The table with the countries, year of the survey, level of poverty and age group shares in population and among MPI poor, can be found at the end of this note.

\section{MORE ON THE OPHI WEBSITE}

For more information, visit the Global MPI Interactive Databank at www.ophi.org.uk/multidimensionalpoverty-index. 
First, we found that there seems to be a relationship between multidimensional poverty and the age structure of a country. Figure 1 plots the population shares of different age groups in the population for our set of countries. ${ }^{\text {iii }}$ The countries are ordered from the one with lowest poverty level, Serbia, to the one with the highest level, Niger. The figure shows that countries with highest levels of MPI tend to have a higher proportion of children under 10 years old, and a lower share of adults across the population. This probably reflects that poorer countries are in earlier stages of demographic transition.
Figure 2 shows the percentage of people who are poor and who belong to each age group. Although there is variation, the gap between the share of adults and the share of children under 10 among the poor tends to be smaller in poorer countries. In Niger and Uganda, children under 10 are the age group with the highest share among the poor, representing around 40 percent of the MPI poor.
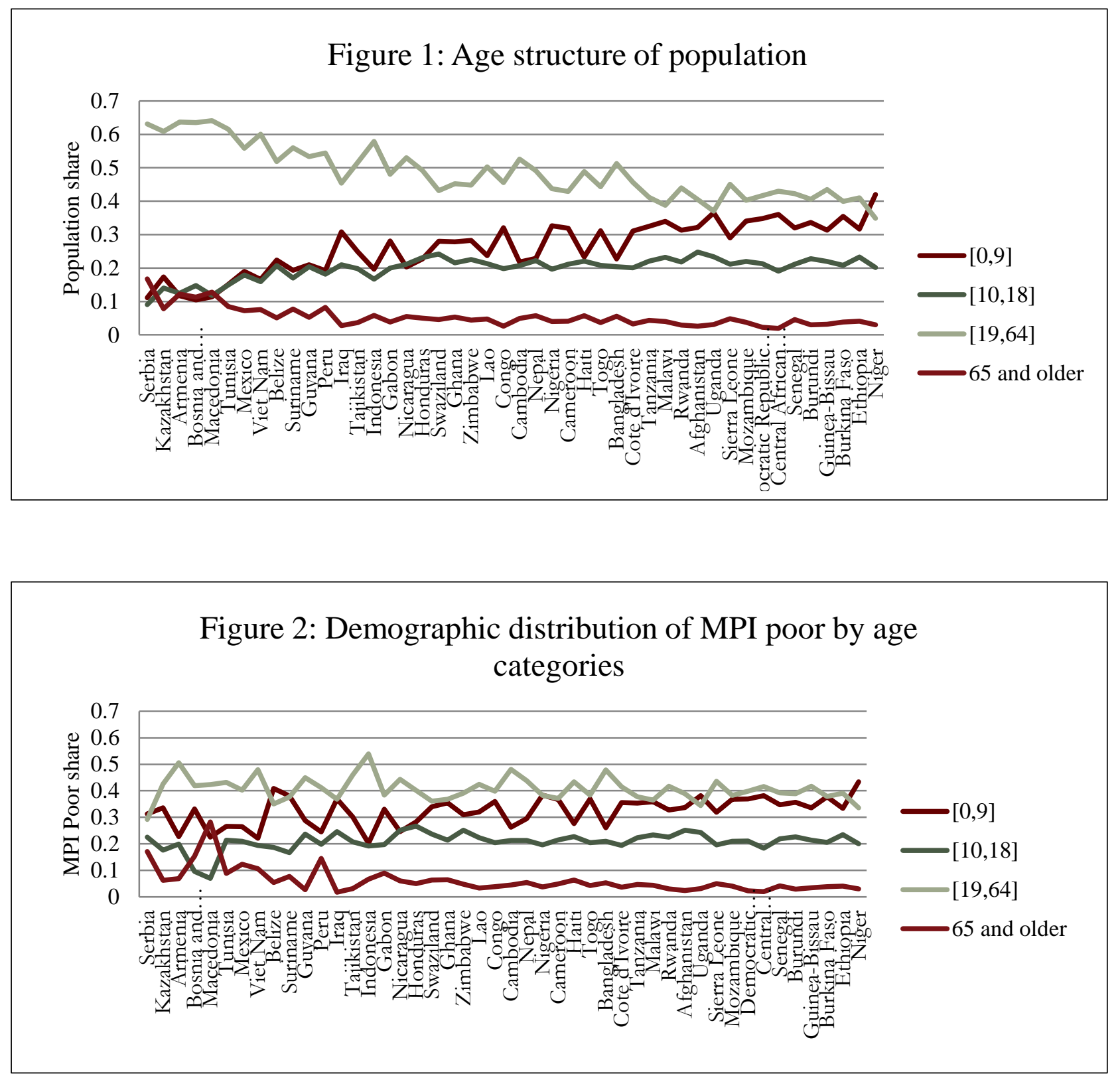
Combining the information from the two graphs above and focusing on the children under 10 (Figure 3), we find that in all countries analyzed, children are over-represented among the poor. This is due to the fact that in practically all countries the average number and share of children under 10 years old is higher in poor households than in non-poor households. ${ }^{\text {iv }}$ The extent of the over-representation seems to be related to the level of poverty. In countries with lower levels of poverty the concentration of children in poor households seems to be higher.
These findings suggest that children are especially likely to be poor: first, because they mostly live in countries with higher levels of poverty; second, because they mostly live in poor households.

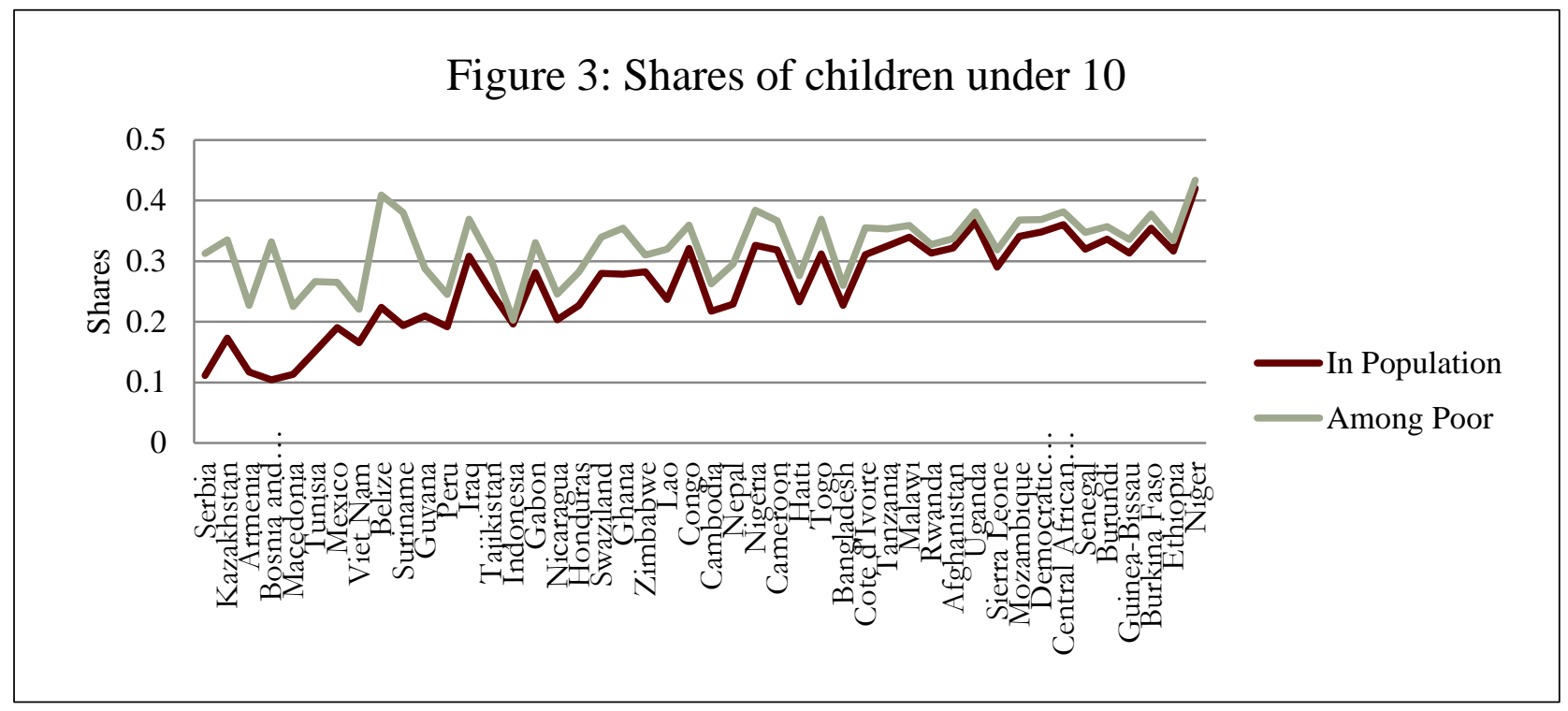

\section{REFERENCES}

Alkire, S. and Roche, J. M. (2012). 'Beyond Headcount: Measures that Reflect the Breadth and Components of Child Poverty', in A. Minujin and S. Nandy (eds.), Global Child Poverty and Well-Being: Measurement, Concepts, Policy and Action. Bristol: The Policy Press.

Alkire, S. and Santos, M. E. (2014). 'Measuring Acute Poverty in the Developing World: Robustness and Scope of the Multidimensional Poverty Index'. World Development, 59: 251-274.

Alkire, S., Conconi, A. and Seth, S. (2014)

Multidimensional Poverty Index: 2014: Brief

Methodological Note and Results. Oxford Poverty and Human Development Initiative (OPHI) Briefing 19.

De Neubourg, C., Chai, J., de Milliano, M., Plavgo, I., and Wei, Z. (2012). 'Step by Step Guidelines to the Multiple Deprivation Analysis (MODA) for Children'. UNICEF Research Working Paper WP-2012-10.

Roche, J. M. (2013). 'Monitoring Progress in Child Poverty Reduction: Methodological Insights and Illustration to the Case Study of Bangladesh'. Social Indicators Research 112(2): 363-390.

\section{ENDNOTES}

i See De Neubourg et al. (2012), Alkire and Roche (2012), and Roche (2013).

ii See Alkire and Santos (2014) for a discussion of the relationship between the size and composition of a household, and its probability of being identified as poor.

iii The population shares were computed using the same survey and sample that was used to compute the Global MPI.

iv In all countries the average number of children under 10 is always higher in poor households than in nonpoor households. This difference, however, is not statistically significant in Serbia and Macedonia. The average share of children under 10 is also higher in poor households than in non-poor households in all countries except Indonesia.

Oxford Poverty \& Human Development Initiative (OPHI) Oxford Department of International Development (ODID)

Queen Elizabeth House (QEH)

University of Oxford, Mansfield Road

Oxford OX1 3TB UK

Telephone: +44 (0)1865 271915

Email: ophi@qeh.ox.ac.uk 


\section{Table Distribution of MPI Poor by Age Group}

The table presents the age groups' shares in populationand among the poor people for 46 countries. These countries are sorted by low to high according to the OPHI's Multidimensional Poverty Index.

\begin{tabular}{|c|c|c|c|c|c|c|c|c|c|c|c|c|c|c|c|c|}
\hline \multirow{3}{*}{ Country } & \multirow{2}{*}{\multicolumn{2}{|c|}{ MPI data source }} & \multicolumn{2}{|c|}{ Multidimensional poverty } & \multirow{2}{*}{\multicolumn{4}{|c|}{$\begin{array}{l}\text { Share of age groups in populatior } \\
{[0 ; 10[\quad[10 ; 19[\quad[19 ; 65[\quad[65 ; \ldots[}\end{array}$}} & \multirow{2}{*}{\multicolumn{4}{|c|}{ re of age groups among poor pec }} & \multirow{2}{*}{\multicolumn{2}{|c|}{$\begin{array}{l}\text { Average no. of household } \\
\text { members aged under } 10\end{array}$}} & \multirow{2}{*}{\multicolumn{2}{|c|}{$\begin{array}{l}\text { Share of no. of household } \\
\text { members aged under } 10\end{array}$}} \\
\hline & & & \multirow{2}{*}{$\begin{array}{c}\begin{array}{c}\text { Multidimensional } \\
\text { Poverty Index } \\
(\mathbf{M P I}=\mathbf{H} * \mathbf{A})\end{array} \\
\text { Range } 0 \text { to } 1\end{array}$} & \multirow{2}{*}{$\begin{array}{c}\begin{array}{c}\text { Headcount } \\
\text { Ratio } \\
\text { (H) }\end{array} \\
\%\end{array}$} & & & & & & & & & & & & \\
\hline & Survey & Year & & & $\%$ & & $\%$ & & & & $\%$ & $\%$ & $\begin{array}{l}\text { Non-poor } \\
\text { households }\end{array}$ & $\begin{array}{c}\text { Poor } \\
\text { households }\end{array}$ & $\begin{array}{l}\text { Non-poor } \\
\text { households }\end{array}$ & $\begin{array}{c}\text { Poor } \\
\text { households }\end{array}$ \\
\hline Serbia & MICS & 2010 & 0.000 & $0.1 \%$ & $11.1 \%$ & $9.0 \%$ & $63.1 \%$ & $16.7 \%$ & $31.3 \%$ & $22.5 \%$ & $29.2 \%$ & $17.0 \%$ & 0.36 & 1.15 & $7.6 \%$ & $14.1 \%$ \\
\hline Kazakhstan & MICS & 2011 & 0.001 & $0.2 \%$ & $17.3 \%$ & $14.0 \%$ & $60.9 \%$ & $7.8 \%$ & $33.5 \%$ & $17.6 \%$ & $42.6 \%$ & $6.3 \%$ & 0.59 & 1.93 & $12.1 \%$ & $27.5 \%$ \\
\hline Armenia & DHS & 2010 & 0.001 & $0.3 \%$ & $11.7 \%$ & $12.4 \%$ & $63.7 \%$ & $12.2 \%$ & $22.7 \%$ & $19.9 \%$ & $50.6 \%$ & $6.9 \%$ & 0.41 & 1.26 & $7.9 \%$ & $22.0 \%$ \\
\hline Bosnia and Herzegovina & MICS & 2012 & 0.002 & $0.5 \%$ & $10.4 \%$ & $14.7 \%$ & $63.5 \%$ & $11.3 \%$ & $33.2 \%$ & $9.6 \%$ & $41.9 \%$ & $15.3 \%$ & 0.36 & 1.34 & $7.9 \%$ & $24.7 \%$ \\
\hline Macedonia & MICS & 2011 & 0.002 & $0.7 \%$ & $11.3 \%$ & $11.8 \%$ & $64.1 \%$ & $12.8 \%$ & $22.5 \%$ & $6.9 \%$ & $42.3 \%$ & $28.2 \%$ & 0.41 & 0.65 & $8.2 \%$ & $13.1 \%$ \\
\hline Tunisia & MICS & 2012 & 0.004 & $1.2 \%$ & $15.1 \%$ & $14.9 \%$ & $61.5 \%$ & $8.5 \%$ & $26.6 \%$ & $21.4 \%$ & $43.2 \%$ & $8.8 \%$ & 0.62 & 1.10 & $13.1 \%$ & $20.2 \%$ \\
\hline Mexico & ENSANUT & 2012 & 0.011 & $2.8 \%$ & $19.0 \%$ & $17.9 \%$ & $55.8 \%$ & $7.2 \%$ & $26.5 \%$ & $20.9 \%$ & $40.3 \%$ & $12.3 \%$ & 0.73 & 0.98 & $15.4 \%$ & $18.6 \%$ \\
\hline Viet Nam & MICS & 2011 & 0.017 & $4.2 \%$ & $16.6 \%$ & $15.9 \%$ & $60.0 \%$ & $7.5 \%$ & $22.1 \%$ & $19.4 \%$ & $48.0 \%$ & $10.6 \%$ & 0.62 & 0.81 & $14.1 \%$ & $16.7 \%$ \\
\hline Belize & MICS & 2011 & 0.018 & $4.6 \%$ & $22.4 \%$ & $20.7 \%$ & $51.8 \%$ & $5.0 \%$ & $40.9 \%$ & $18.6 \%$ & $35.1 \%$ & $5.4 \%$ & 0.82 & 2.17 & $16.1 \%$ & $30.6 \%$ \\
\hline Suriname & MICS & 2010 & 0.024 & $5.9 \%$ & $19.3 \%$ & $17.0 \%$ & $56.0 \%$ & $7.7 \%$ & $38.0 \%$ & $16.6 \%$ & $37.7 \%$ & $7.7 \%$ & 0.69 & 1.51 & $13.3 \%$ & $29.4 \%$ \\
\hline Guyana & DHS & 2009 & 0.030 & $7.7 \%$ & $21.0 \%$ & $20.4 \%$ & $53.4 \%$ & $5.3 \%$ & $28.8 \%$ & $23.6 \%$ & $44.9 \%$ & $2.7 \%$ & 0.76 & 1.39 & $15.4 \%$ & $22.8 \%$ \\
\hline Peru & DHS-Cont & 2012 & 0.043 & $10.5 \%$ & $19.2 \%$ & $18.1 \%$ & $54.4 \%$ & $8.2 \%$ & $24.5 \%$ & $19.8 \%$ & $41.2 \%$ & $14.5 \%$ & 0.71 & 0.83 & $15.1 \%$ & $15.3 \%$ \\
\hline Iraq & MICS & 2011 & 0.045 & $11.6 \%$ & $30.8 \%$ & $21.0 \%$ & $45.4 \%$ & $2.8 \%$ & $36.9 \%$ & $24.6 \%$ & $36.8 \%$ & $1.7 \%$ & 1.91 & 3.16 & $28.5 \%$ & $37.3 \%$ \\
\hline Tajikistan & DHS & 2012 & 0.054 & $13.2 \%$ & $24.9 \%$ & $19.8 \%$ & $51.6 \%$ & $3.7 \%$ & $30.1 \%$ & $20.7 \%$ & $46.0 \%$ & $3.1 \%$ & 1.47 & 2.46 & $21.2 \%$ & $28.2 \%$ \\
\hline Indonesia & DHS & 2012 & 0.066 & $15.5 \%$ & $19.6 \%$ & $16.7 \%$ & $57.9 \%$ & $5.8 \%$ & $20.2 \%$ & $19.1 \%$ & $53.9 \%$ & $6.7 \%$ & 0.78 & 0.82 & $16.9 \%$ & $16.0 \%$ \\
\hline Gabon & DHS & 2012 & 0.070 & $16.5 \%$ & $28.1 \%$ & $20.0 \%$ & $48.0 \%$ & $3.9 \%$ & $33.1 \%$ & $19.7 \%$ & $38.4 \%$ & $8.9 \%$ & 1.06 & 1.63 & $19.2 \%$ & $22.8 \%$ \\
\hline Nicaragua & DHS & 2012 & 0.072 & $16.1 \%$ & $20.3 \%$ & $21.1 \%$ & $53.1 \%$ & $5.5 \%$ & $24.6 \%$ & $25.1 \%$ & $44.3 \%$ & $6.0 \%$ & 0.87 & 1.14 & $17.2 \%$ & $21.0 \%$ \\
\hline Honduras & DHS & 2012 & 0.072 & $15.8 \%$ & $22.7 \%$ & $23.1 \%$ & $49.2 \%$ & $5.0 \%$ & $28.2 \%$ & $26.7 \%$ & $40.1 \%$ & $5.0 \%$ & 0.94 & 1.43 & $18.9 \%$ & $24.3 \%$ \\
\hline Swaziland & MICS & 2010 & 0.086 & $20.4 \%$ & $28.0 \%$ & $24.2 \%$ & $43.2 \%$ & $4.6 \%$ & $33.9 \%$ & $23.7 \%$ & $36.1 \%$ & $6.3 \%$ & 1.10 & 1.62 & $19.8 \%$ & $26.9 \%$ \\
\hline Ghana & MICS & 2011 & 0.139 & $30.4 \%$ & $27.9 \%$ & $21.5 \%$ & $45.2 \%$ & $5.4 \%$ & $35.4 \%$ & $21.4 \%$ & $36.8 \%$ & $6.4 \%$ & 0.88 & 1.66 & $18.2 \%$ & $28.9 \%$ \\
\hline Zimbabwe & DHS & 2011 & 0.172 & $39.1 \%$ & $28.2 \%$ & $22.6 \%$ & $44.8 \%$ & $4.4 \%$ & $31.0 \%$ & $25.1 \%$ & $39.1 \%$ & $4.8 \%$ & 1.01 & 1.48 & $21.8 \%$ & $27.6 \%$ \\
\hline
\end{tabular}




\begin{tabular}{|c|c|c|c|c|c|c|c|c|c|c|c|c|c|c|c|c|}
\hline \multirow{4}{*}{ Country } & \multirow{2}{*}{\multicolumn{2}{|c|}{ MPI data source }} & \multicolumn{2}{|c|}{ Multidimensional poverty } & \multirow{3}{*}{\multicolumn{4}{|c|}{$\begin{array}{l}\text { Share of age groups in populatior } \\
{[0 ; 10[\quad[10 ; 19[\quad[19 ; 65[\quad[65 ; \ldots[}\end{array}$}} & \multirow{2}{*}{\multicolumn{4}{|c|}{ re of age groups among poor pec }} & \multirow{2}{*}{\multicolumn{2}{|c|}{$\begin{array}{l}\text { Average no. of household } \\
\text { members aged under } 10\end{array}$}} & \multirow{2}{*}{\multicolumn{2}{|c|}{$\begin{array}{c}\text { Share of no. of household } \\
\text { members aged under } 10\end{array}$}} \\
\hline & & & \multirow{3}{*}{$\begin{array}{c}\begin{array}{c}\text { Multidimensional } \\
\text { Poverty Index } \\
\left(\mathbf{M P I}=\mathbf{H}^{* \mathbf{A}}\right)\end{array} \\
\text { Range } 0 \text { to } 1\end{array}$} & \multirow{3}{*}{$\begin{array}{c}\begin{array}{c}\text { Headcount } \\
\text { Ratio } \\
(\mathbf{H})\end{array} \\
\%\end{array}$} & & & & & & & & & & & & \\
\hline & \multirow{2}{*}{ Survey } & \multirow{2}{*}{ Year } & & & & & & & & & & & \multirow{2}{*}{$\begin{array}{l}\text { Non-poor } \\
\text { households }\end{array}$} & \multirow{2}{*}{$\begin{array}{c}\text { Poor } \\
\text { households }\end{array}$} & \multirow{2}{*}{$\begin{array}{l}\text { Non-poor } \\
\text { households }\end{array}$} & \multirow{2}{*}{$\begin{array}{c}\text { Poor } \\
\text { households }\end{array}$} \\
\hline & & & & & $\%$ & $\%$ & $\%$ & $\%$ & $\%$ & $\%$ & $\%$ & $\%$ & & & & \\
\hline Lao & MICS/DHS & 2012 & 0.174 & $34.1 \%$ & $23.7 \%$ & $21.3 \%$ & $50.3 \%$ & $4.8 \%$ & $32.0 \%$ & $22.3 \%$ & $42.4 \%$ & $3.3 \%$ & 0.94 & 1.88 & $17.6 \%$ & $29.4 \%$ \\
\hline Congo & DHS & 2012 & 0.181 & $39.7 \%$ & $32.1 \%$ & $19.8 \%$ & $45.5 \%$ & $2.6 \%$ & $36.0 \%$ & $20.4 \%$ & $39.9 \%$ & $3.8 \%$ & 1.24 & 1.76 & $24.0 \%$ & $29.6 \%$ \\
\hline Cambodia & DHS & 2010 & 0.212 & $45.9 \%$ & $21.8 \%$ & $20.8 \%$ & $52.6 \%$ & $4.9 \%$ & $26.3 \%$ & $21.2 \%$ & $48.1 \%$ & $4.4 \%$ & 0.85 & 1.24 & $16.4 \%$ & $24.1 \%$ \\
\hline Nepal & DHS & 2011 & 0.217 & $44.2 \%$ & $22.9 \%$ & $22.2 \%$ & $49.2 \%$ & $5.7 \%$ & $29.5 \%$ & $21.3 \%$ & $43.8 \%$ & $5.3 \%$ & 0.74 & 1.35 & $15.7 \%$ & $25.6 \%$ \\
\hline Nigeria & MICS & 2011 & 0.240 & $43.3 \%$ & $32.7 \%$ & $19.6 \%$ & $43.7 \%$ & $4.0 \%$ & $38.4 \%$ & $19.6 \%$ & $38.2 \%$ & $3.7 \%$ & 1.28 & 2.22 & $23.4 \%$ & $32.7 \%$ \\
\hline Cameroon & DHS & 2011 & 0.248 & $46.0 \%$ & $31.9 \%$ & $21.2 \%$ & $42.9 \%$ & $4.1 \%$ & $36.7 \%$ & $21.4 \%$ & $37.1 \%$ & $4.8 \%$ & 1.29 & 2.14 & $21.3 \%$ & $30.3 \%$ \\
\hline Haiti & DHS & 2012 & 0.248 & $49.4 \%$ & $23.3 \%$ & $22.1 \%$ & $48.9 \%$ & $5.8 \%$ & $27.6 \%$ & $22.7 \%$ & $43.4 \%$ & $6.3 \%$ & 0.82 & 1.22 & $16.0 \%$ & $22.1 \%$ \\
\hline Togo & MICS & 2010 & 0.250 & $49.8 \%$ & $31.2 \%$ & $20.8 \%$ & $44.3 \%$ & $3.7 \%$ & $36.9 \%$ & $20.4 \%$ & $38.4 \%$ & $4.3 \%$ & 1.11 & 1.96 & $20.9 \%$ & $31.5 \%$ \\
\hline Bangladesh & DHS & 2011 & 0.253 & $51.3 \%$ & $22.7 \%$ & $20.4 \%$ & $51.3 \%$ & $5.6 \%$ & $26.0 \%$ & $20.9 \%$ & $47.8 \%$ & $5.2 \%$ & 0.87 & 1.21 & $17.7 \%$ & $23.8 \%$ \\
\hline Cote d'Ivoire & DHS & 2012 & 0.310 & $58.7 \%$ & $31.0 \%$ & $20.0 \%$ & $45.6 \%$ & $3.3 \%$ & $35.5 \%$ & $19.3 \%$ & $41.5 \%$ & $3.6 \%$ & 1.14 & 1.97 & $19.2 \%$ & $29.6 \%$ \\
\hline Tanzania & DHS & 2010 & 0.332 & $65.6 \%$ & $32.5 \%$ & $22.1 \%$ & $41.1 \%$ & $4.3 \%$ & $35.3 \%$ & $22.3 \%$ & $37.7 \%$ & $4.7 \%$ & 1.19 & 1.89 & $22.4 \%$ & $30.2 \%$ \\
\hline Malawi & DHS & 2010 & 0.334 & $66.7 \%$ & $34.0 \%$ & $23.2 \%$ & $38.8 \%$ & $4.0 \%$ & $35.9 \%$ & $23.4 \%$ & $36.5 \%$ & $4.3 \%$ & 1.30 & 1.70 & $26.3 \%$ & $31.5 \%$ \\
\hline Rwanda & DHS & 2010 & 0.350 & $69.0 \%$ & $31.3 \%$ & $21.8 \%$ & $44.0 \%$ & $3.0 \%$ & $32.8 \%$ & $22.5 \%$ & $41.7 \%$ & $3.0 \%$ & 1.20 & 1.48 & $24.1 \%$ & $28.9 \%$ \\
\hline Afghanistan & MICS & 2011 & 0.353 & $66.2 \%$ & $32.2 \%$ & $24.8 \%$ & $40.5 \%$ & $2.6 \%$ & $33.7 \%$ & $25.1 \%$ & $38.9 \%$ & $2.3 \%$ & 2.18 & 2.67 & $28.0 \%$ & $33.1 \%$ \\
\hline Uganda & DHS & 2011 & 0.367 & $69.9 \%$ & $36.5 \%$ & $23.4 \%$ & $37.0 \%$ & $3.1 \%$ & $38.2 \%$ & $24.3 \%$ & $34.4 \%$ & $3.1 \%$ & 1.34 & 1.98 & $25.6 \%$ & $33.2 \%$ \\
\hline Sierra Leone & MICS & 2010 & 0.388 & $72.5 \%$ & $29.0 \%$ & $21.1 \%$ & $45.1 \%$ & $4.8 \%$ & $31.8 \%$ & $19.5 \%$ & $43.6 \%$ & $5.0 \%$ & 1.17 & 1.92 & $18.7 \%$ & $30.3 \%$ \\
\hline Mozambique & DHS & 2011 & 0.389 & $69.6 \%$ & $34.1 \%$ & $21.9 \%$ & $40.2 \%$ & $3.7 \%$ & $36.8 \%$ & $21.0 \%$ & $38.2 \%$ & $4.0 \%$ & 1.22 & 1.60 & $23.0 \%$ & $30.2 \%$ \\
\hline Democratic Republic of Congc & MICS & 2010 & 0.392 & $74.0 \%$ & $34.8 \%$ & $21.3 \%$ & $41.7 \%$ & $2.3 \%$ & $36.9 \%$ & $21.1 \%$ & $39.8 \%$ & $2.3 \%$ & 1.56 & 1.96 & $25.0 \%$ & $33.1 \%$ \\
\hline Central African Republic & MICS & 2010 & 0.430 & $77.6 \%$ & $36.1 \%$ & $19.0 \%$ & $43.0 \%$ & $1.9 \%$ & $38.1 \%$ & $18.3 \%$ & $41.6 \%$ & $1.9 \%$ & 1.36 & 1.82 & $23.9 \%$ & $32.1 \%$ \\
\hline Senegal & DHS & 2011 & 0.439 & $74.4 \%$ & $32.0 \%$ & $21.2 \%$ & $42.2 \%$ & $4.6 \%$ & $34.7 \%$ & $21.9 \%$ & $39.2 \%$ & $4.2 \%$ & 1.78 & 3.67 & $20.7 \%$ & $32.7 \%$ \\
\hline Burundi & DHS & 2010 & 0.454 & $80.8 \%$ & $33.7 \%$ & $22.8 \%$ & $40.6 \%$ & $3.0 \%$ & $35.7 \%$ & $22.6 \%$ & $38.9 \%$ & $2.9 \%$ & 1.18 & 1.72 & $21.9 \%$ & $31.5 \%$ \\
\hline Guinea-Bissau & MICS & 2006 & 0.462 & $77.5 \%$ & $31.3 \%$ & $22.0 \%$ & $43.5 \%$ & $3.2 \%$ & $33.6 \%$ & $21.3 \%$ & $41.7 \%$ & $3.4 \%$ & 1.74 & 2.67 & $23.3 \%$ & $32.6 \%$ \\
\hline Burkina Faso & DHS & 2010 & 0.535 & $84.0 \%$ & $35.5 \%$ & $20.8 \%$ & $39.9 \%$ & $3.8 \%$ & $37.7 \%$ & $20.5 \%$ & $37.9 \%$ & $3.8 \%$ & 1.07 & 2.22 & $19.7 \%$ & $33.3 \%$ \\
\hline Ethiopia & DHS & 2011 & 0.564 & $87.3 \%$ & $31.7 \%$ & $23.3 \%$ & $41.0 \%$ & $4.0 \%$ & $33.4 \%$ & $23.4 \%$ & $39.1 \%$ & $4.0 \%$ & 0.69 & 1.63 & $14.9 \%$ & $29.3 \%$ \\
\hline Niger & DHS & 2012 & 0.605 & $89.3 \%$ & $42.0 \%$ & $20.2 \%$ & $34.8 \%$ & $3.0 \%$ & $43.4 \%$ & $20.0 \%$ & $33.6 \%$ & $3.0 \%$ & 1.52 & 2.62 & $26.0 \%$ & $39.1 \%$ \\
\hline
\end{tabular}

